\title{
A blended user centred design study for wearable haptic gait rehabilitation following hemiparetic stroke
}

\author{
Theodoros Georgiou ${ }^{1}$, Simon Holland ${ }^{1}$, Janet van der Linden ${ }^{1}$, Josie Tetley ${ }^{2}$, Rachel C. Stockley ${ }^{2}$, Glenis Donaldson ${ }^{2}$, \\ Linda Garbutt ${ }^{2}$, Ornella Pinzone ${ }^{2}$, Fanny Grasselly ${ }^{1}$, Kevin Deleaye $^{1}$
}

\author{
Centre for Research in Computing ${ }^{1}$ \\ The Open University \\ Milton Keynes, MK7 6AA, UK \\ Theodoros.Georgiou@open.ac.uk
}

\begin{abstract}
Restoring mobility and rehabilitation of gait are high priorities for post-stroke rehabilitation. Cueing using metronomic rhythmic sensory stimulation has been shown to improve gait, but most versions of this approach have used auditory and visual cues. In contrast, we developed a prototype wearable system for rhythmic cueing based on haptics, which was shown to be highly effective in an early pilot study. In this paper we describe a follow-up study with four stroke survivors to inform design, and to identify issues and requirements for such devices to be used in home-based or out-door settings. To this end, we present a blended user-centred design study of a wearable haptic gait rehabilitation system. This study draws on the combined views of physiotherapists, nurses, interaction designers and stroke survivors. Many of the findings were unanticipated, identifying issues outside the scope of initial designs, with important implications for future design and appropriate use.
\end{abstract}

Keywords- blended user centred design; haptic bracelets; stroke; gait rehabilitation; walking; tactile metronome; hemiparetic; haptic metronome; entrainment

\section{INTRODUCTION}

Stroke is a serious, sudden and devastating illness, affecting approximately fifteen million people worldwide each year [1]. In the UK alone there are 1.1 million stroke survivors and over one hundred thousand new strokes occur every year; more than one every five minutes [2]. This makes stroke one of the leading causes of adult disabilities [3], leaving more than half of all stroke survivors dependent on others for every day activities [4].

Following a stroke, after acute specialist hospital care, regular rehabilitation exercises can significantly improve a person's recovery both in the early days after a stroke and long after they return home [5]. However exercising in the home setting without guidance can be difficult to carry out effectively.

Recent advancements in technology create the possibility of small, light inconspicuous devices, capable of supporting dayto-day rehabilitation exercises by providing appropriate guidance and monitoring. However, there are serious challenges involved in the design of such systems. New health care technologies can raise challenging issues of design and appropriate usage. Wearable healthcare systems need to take into account physical, sensory and cognitive abilities of the intended users of the system. Addressing these problems

\author{
Faculty of Health, Psychology and Social Care ${ }^{2}$ \\ Manchester Metropolitan University \\ Manchester, M15 6GX, UK
}

involves user-centred approaches. These typically involve building prototypes with which users can engage with in depth and reflect on. Prototypes also help designers to understand diverse real world issues and to identify and evaluate potential improvements. They enable participants to physically explore the possibilities of new technologies, and provide a source of inspiration for future use scenarios. In [6] such a design approach is referred to as a blended design approach, combining technology inspiration, user-centred design and consulting with experts.

In this paper we describe our findings from conducting such blended design sessions for stroke survivors. Our study focuses on gait rehabilitation through a technology known as the Haptic Bracelets, which provide haptic (i.e. touch-based) cueing, sensing and communication [7], [8]. The Haptic Bracelets (discussed in detail below) were originally developed to support the development of musical skills in learning multi-limbed rhythms [9], [10]. They were subsequently adapted for gait rehabilitation using haptic cues, drawing on the literature and the successful use of rhythmic sensory cueing for gait rehabilitation using audio and visual cues [11] [12] [13] [14] [15].

An initial pilot study with a stroke survivor using the Haptic Bracelets to provide rhythmic sensory haptic cues was promising and showed immediate improvements in gait and increased flexion at the knee [7], [16]. The present study is aimed at exploring the wider physical, sensory and cognitive issues needed to develop the system for wider use in rehabilitation outside the lab, through collaboration between interaction designers, physiotherapists, rehabilitation experts, and stroke survivors.

\section{RELATED WORK}

\section{A. Hemiparetic Gait in Stroke Survivors}

About four out of five stroke victims survive their stroke [2], but experience "hemiparetic gait", a condition characterised variously by reduced walking speed [17], stride time variability [18], increased step length variability [18], and temporal and spatial gait asymmetry [19]. Many health problems are associated with this disorder, for example the non-paretic (stronger) limb may be exposed to higher vertical forces [20] which can lead to joint pains [21], degeneration [22] and 
increased risk of fractures. Hemiparetic gait is directly linked to an increased risk of falling observed after stroke, doubling the risk of hip fracture [23]. Besides the physical health issues, gait rehabilitation is also of paramount importance for the restoration of independence and thus an overall better quality of life [24].

\section{B. Audio, Visual and Haptic Support for Gait Rehabilitation}

In order to support gait rehabilitation for people suffering from various neurological conditions, researchers have explored the idea of metronomic cueing using a variety of sensory channels, principally auditory and visual.

Use of an auditory rhythm provided by a metronome has been investigated and successfully demonstrated as a means of improving hemiparetic gait with immediate, though not lasting, effects [11]. Studies where participants were asked to walk on a treadmill showed that they could synchronise their steps to a rhythmic audio metronome [12]. Having an audio cue to provide the rhythm for walking also helped participants with post-stroke gait impairments to show improvements in spatial [13] and temporal symmetry [12]. The step time asymmetry and the paretic step time variability of participants also significantly improved [14], as was the ability to make gait adjustments in response to changes in the cue [15]. Rhythmic cueing is therefore a promising approach, but the use of audio may not be the best medium for the in-home and out-and-about scenarios for rehabilitation where it is important to keep the audio channel clear in order to be able to remain aware of the environment, oncoming traffic or conversations with other people.

Studies with healthy elderly participants walking on treadmill indicate that visual cues were more effective in changing the pattern of gait than an audio metronome [25]. Visual walking cues in the form of fixed markers on the floor, (e.g., stripes painted on the floor or virtual stepping markers), have been reported to have a number of benefits in gait rehabilitation of patients with neurological conditions affecting their gait. In studies with people affected by Parkinson's disease, visual markers projected on the floor show an increase in stride length and walking velocity [26]. On treadmill walking with healthy elderly participants, visual spatial cues in the form of projected stepping-stones also show promise [25]. However, approaches involving visual cues and projections in front of a user while walking can be intrusive and do not easily translate to home based scenarios as they typically require extensive laboratory installations.

A recent pilot study [8] using Haptic Bracelets to provide vibrotactile rhythmic cueing for post-stroke gait rehabilitation demonstrated immediate benefits similar to auditory cueing. With tactile cueing, step length was found to increase, and a range of other measures: paretic, hip angle at toe off, peak knee flexion during swing and ankle range of motion all increased beneficially. The participant noted that the cueing "helps me to walk in time" and "helps me to stand up straight and walk properly" [8].

\section{Entrainment vs Stimulus Response}

When designing for rhythmic sensory cueing in general, whether by auditory, visual or haptic means, the theory of sensory motor entrainment [9] [10] [16] provides the most appropriate framework for understanding and analysis.

Entrainment is the term used by psychologists to describe the sensory motor capabilities that allow humans to synchronise their movements exactly to a regular beat perceived in any sensory modality (auditory, visual or tactile). This capability has been found to apply to periodicities in the range of about $200 \mathrm{~ms}$ to 2 seconds [16] [27]. Aspects of this facility are limited to humans and certain species of bird, yet have been demonstrated to be present in human neonates [28]. For details of the postulated neural mechanisms behind sensory motor entrainment, see [27].

Entrainment is the common process behind the various applications of metronomic rhythmic sensory cueing in any modality. It is important to clearly distinguish between entrainment and the contrasting process of stimulus response. To understand the difference, consider everyday applications of vibrotactiles, which typically focus on notifications and alerts. Applications of this kind are best understood in terms of stimulus response, as follows. When, for example, a smartphone in silent mode vibrates to give an alert, there is a necessary delay in perception while the sensory stimulus is processed, and then a further delay while any resulting human action is enacted. Broadly, this process is one of stimulus and response (though variants can involve cognitively learned responses, conditioned responses, or direct muscle responses an issue we will return to below).

Thus, responses to notifications and alerts always involve delays. By contrast, after hearing a few initial beats, most people can generally tap along to a regular pulse in more or less exact synchronisation. In the case of rhythmic sensory cueing in any modality for gait rehabilitation, the key instruction for users is to "time your footfalls to the beat". Thus, entrainment is the common foundation for the various applications of metronomic rhythmic sensory stimulation in any modality.

\section{Rapid prototyping and technological inspiration}

When building wearable systems which must fit into everyday life, a valuable approach is to use rapid prototyping, also referred to as 'sketching in hardware', or 'physical computing' [29]. This involves building physical, working (though sometimes incomplete) prototypes for representative users to interact with. Hardware prototypes differ from paperbased prototypes, by providing working functionality. Prototypes often dispense with an aesthetically pleasing appearance, and may be assembled using a variety of DIY techniques such as hardware hacking and 3D printing. Rapid prototyping typically goes through cycles, where user reactions feed into the next iteration of the prototype.

Apart from helping the users understand new approaches, prototyping is also important for the designers of a system, because building the physical system can be a uniquely informative way of learning about the affordances of, and constraints on, systems. [30]. Understanding the potential of technologies, by physically exploring them through hands-on engagement can provide valuable inspiration for new designs. Rogers et al. [31] use the term 'technology inspired' to describe the process where the capabilities of new technologies are 


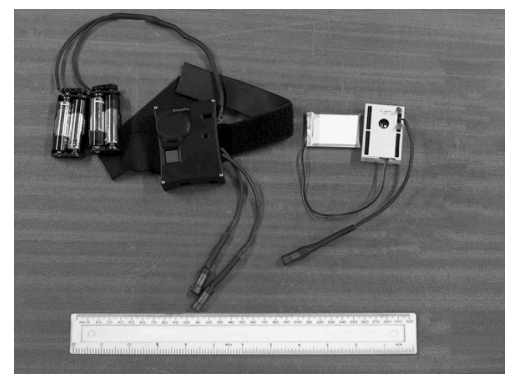

Fig. 1. A current Haptic Bracelet (right) next to the older and larger Haptic Bracelets (left). For technical reasons, both versions were worn together in the current study (see text)

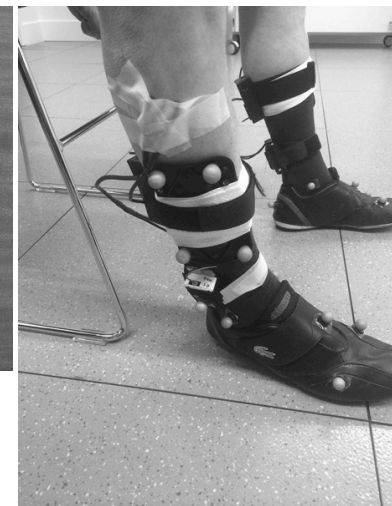
Bracelets and optical tracking markers (see text)
Fig. 2. Participant wearing Haptic

explored and experimented with, in order to provide ideas for new conceptual development and interaction design.

\section{STUDY Aims}

The principal aim of the present study is to investigate design issues in the use of haptic technology for rhythmic sensory stimulation as a way of providing gait rehabilitation for stroke survivors. In particular we aim to begin to identify issues involved in transferring the approach from the lab to selfmanaged use at home or outdoors.

\section{METHODOLOGY}

The approach taken was that of a blended design session. This involved gathering diverse views from stroke survivors, designers and contributing health professionals while actively engaging with a rehabilitation procedure using the Haptic Bracelets. The interdisciplinary research team included a professor of nursing, two experienced physiotherapists, three interaction designers and a motion capture technician. The setting was that of a newly installed university laboratory, specifically set up for the study of gait rehabilitation. The session lasted three days - with each stroke survivor present for two of the three days while spending a considerable amount of time with our interdisciplinary research team. Participants were helped to fit the Haptic Bracelets, and at various times walked both cued and un-cued in a straight line while being videoed and subject to motion capture. Members of the team informally observed and discussed with participants diverse aspects of their experiences.

We held in-depth interviews with each participant at the end of their session, exploring the difficulties they have in their every day life, what needs they might anticipate in respect of the new technology and their initial thoughts on the existing prototype system. At the end of the three-day session we also had a group discussion with the physiotherapists on their views of the technology, discussing issues such as where exactly the technology should be worn, and the point during recovery at which stroke survivors would be most likely to benefit from the technology. Overall, working within such an interdisciplinary group allowed us to gain better insight of the requirements, both from the physiotherapists' and the stroke survivors' perspectives.
TABLE 1 PARTICIPANT DEMOGRAPHICS

\begin{tabular}{|c|c|c|c|c|}
\hline Participant & Gender & Age & Year of stroke & Paretic side \\
\hline P1 & Male & 57 & 2004 & Right \\
\hline P2 & Female & 46 & 2001 & Right \\
\hline P3 & Male & 68 & 1972 & Right \\
\hline P4 & Male & 63 & 2009 & Right \\
\hline
\end{tabular}

\section{A. Technology}

The Haptic Bracelets are a lightweight, wearable wireless technology able to provide rhythmic sensory stimulation via vibrotactiles. Generally one bracelet is worn on each leg, though this depended on survivors' personal preferences, as discussed below. The bracelets are also able to provide highresolution motion capture data for both legs independently. In the present study the motion data was used simply to collect baseline information and give context to user-centred observations: in studies elsewhere, these facilities are used for adaptive cueing [8] and gathering clinical data.

In the present study, we used a new version (figure 1, right) of the Haptic Bracelets [7] [9]. The Haptic Bracelets can deliver haptic pulses via high precision low-latency vibrotactiles with wide dynamic range. The metronomic delivery of pulses to alternate legs is co-ordinated via a central control unit. The current version of the Haptic Bracelets (figure 1, right) is smaller and lighter than earlier versions [7] [9] (figure 1, left) with lower power consumption and richer motion sensing capabilities. Motion sensing is provided by inertial measurement units with three-axis gyros, accelerometers and magnetometers. These feature a sampling rate of around 400 $\mathrm{Hz}$. This fine-grained motion data is communicated to a central control unit via Wi-Fi. The data is time-stamped, logged and used to calculate gait information including cadence and temporal asymmetry. Currently, the central control unit is a laptop, but there are no problems of principle in porting this functionality to a smartphone for applications outside the lab.

For the present trial, as a transitional measure to aid the development of the new version, both older and newer versions of the bracelets were used together (figure 2). Furthermore, for the purposes of collecting baseline information, giving context to user-centred observations, and to assist calibration of the bracelets, we pooled motion capture data from the bracelets and a Qualisys optical motion tracking system.

\section{B. Participants}

The participants for the session were four adults with chronic hemiparesis and aphasia, ranging in age from 46 to 68 who had suffered their strokes some time ago (see Table 1). They had all recovered the ability to speak, although still with some difficulty. All could walk unaided, but not for very long and similarly with some difficulty. They were highly motivated participants who regularly participate in research projects and are much at ease with the physiotherapists, other staff and lab setting. In particular there seemed to be little problem with the Hawthorne effect [32]. All seemed ready to give honest critical opinions and were not shy to do so. Some participants had become good friends over the years as a result of their stroke 
and their participation in various initiatives to support stroke survivors.

\section{Procedure}

The sessions were spread out over three days and were arranged in such a way that participants were not expected to do too much walking in one day. On the first day all participants attended the lab and participated in a standard motion capture session where their gait was recorded using the Qualisys camera system. As noted previously, this data served as baseline for later sessions but also served to get a general sense of their walking speed and rhythm. During this first day the Haptic Bracelet prototype was presented to participants to allow them get an early feel of the new technology and for researchers to get initial feedback on its design and on envisaged potential use in everyday life.

On day 2, the first two of the participants, and on day 3 the second two participants took part in structured hands-on sessions with the technology. At the start of each session, equipment was setup and participants changed from their normal clothes into shorts to allow reflective markers used by the external motion capture system to be clearly placed. The Haptic Bracelets were then strapped on to both legs of each participant with Velcro straps. During the setting up period there were informal conversations with participants, checking that they were comfortable with the set-up. Issues emerging from the exact placement of the vibrotactiles are considered in the findings section below.

\section{1) Walk without cue}

Participants were asked to walk, as they would normally do from a "start" marker on the floor to a "finish" marker at the other side of the room. The length of start to finish was exactly ten meters. Walking from start to finish and then from finish to start counted as two trials. Each participant carried out a number of trials until sufficient baseline kinematic data was collected. A chair was provided at either end for participants to rest whenever they needed.

\section{2) Physically Exploring the Technology}

After completing a number of trials without haptic cueing, the vibrotactile metronome was switched on. Initially, participants were asked simply to sit on a chair and feel the buzzes. The vibrotactile buzz intensity was adjusted so that pulses could be felt clearly but without causing any discomfort.

Once the intensity was set to a comfortable level, the period of the metronome buzz was adjusted for every participant to match his or her walking speed. The baseline measurements taken on day 1 were used as starting points. Taking baseline measurements involved measuring the step cycle (i.e. the average time from heel strike to heel strike for the same leg) and then halving it. For example, if the baseline was calculated to be an average of $1400 \mathrm{~ms}$ for a complete step cycle, then the haptic metronome was set to alternate buzzes between legs every $700 \mathrm{~ms}$.

Once the vibrotactile intensity and the metronome period was adjusted, we asked the participants to stand up and try to step in place following the metronome's rhythm. At this stage we would ask them again if they felt like they needed any further adjustments to be made on the metronome period or the vibrotactile intensity. Throughout this "familiarisation" stage, we would engage in conversation with the participants asking about their initial thoughts and feelings.

3) Walking with cue

When the participants confirmed they were ready to proceed, we asked them to try to walk while trying to "follow the rhythm". We chose not to give very specific instructions to the participants on how to use the metronome in order to allow a

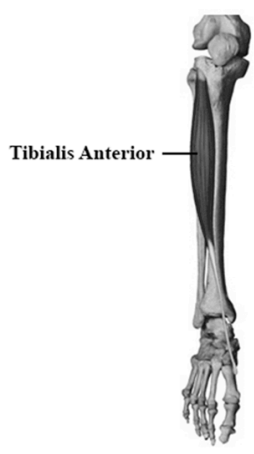

Fig. 3. Location of the Tibialis Anterior muscle. range of behaviours. Participants were asked to walk through the ten-meter long space as they did in the session without the haptic cue.

\section{FINDINGS}

\section{A. Location, strength and timing of cues}

Although we initially suggested to all participants that they wear two Haptic Bracelets (one on each leg), there was some variation in how subjects responded to the suggestion. We explained that this approach would allow each leg to be cued in turn (as well as allowing the bracelets to collect motion data for each leg independently). However, we encouraged each participant to express any alternative preferences they might have. In the event, all participants accepted the initial suggestion, except a single participant (P1), who chose to wear a single bracelet on a single leg. He explained that he found it difficult to switch attention between legs fast enough. Interestingly, he chose to place the vibrotactile on his affected leg, even though this had much less sensitivity (more on this below). He felt that by focusing on that weaker leg it helped him to pay attention to it.

All survivors had a greater or lesser degree of spatial and temporal asymmetry between the affected and non-affected leg. Generally, as one of the physiotherapists later explained, survivors try to spend as little time as possible on their affected side, simply because they "don't trust it enough (sic)".

The exact placement of the vibrotactiles was decided by the physiotherapists. In general, they decided to tape the vibrotactiles on the Tibialis Anterior muscle (see figure 3) using surgical tape. They felt that this placement was most likely to stimulate appropriate movement autonomously. This choice raised questions of balancing the notion of entrainment with elements of stimulus response, following the discussion of this distinction above, and as discussed in detail below.

The part of the Haptic Bracelets being used to gather inertial motion data was strapped near the ankles, on the grounds that this is generally considered a good place for recording movement data. The bony structure of the ankle minimises force absorption and the devices at that place can be strapped in an almost vertical orientation. In some cases where a participant wore a splint, placement had to be adjusted accordingly. See figure 2 for positioning. 
The above observations raise interesting design and therapeutic issues. Rhythmic audio stimulation is known to yield immediate (though not necessarily lasting) improvements to gait (e.g. [11] [12] [13] [14] [15]) through entrainment, and there is good preliminary evidence that rhythmic haptic stimulation can do the same [7], [8]. However, rhythmic haptic stimulation raises further issues in that haptics can be configured specifically to deliver alternate beats to alternate limbs (or all beats to a specifically chosen limb, as noted above). As subsequently emerged in discussion with therapists, this raises questions of how different embodied spatial arrangements for the delivery of rhythmic haptic cues may interact with issues of sensitivity, attention and proprioception. It also raises the question of whether there is potential to use different spatial placement to manipulate attention and proprioception in particular cases for enhanced therapeutic benefit. These issues may also be complicated by the phenomenon of haptic masking: when a limb is subject to impacts, it makes it harder to detect simultaneous touches [9].

The choice by the physiotherapists of placing the vibrotactiles on the Tibialis Anterior muscle represents a line of thought that contrasts with straightforward entrainment, which, after all, can be afforded by using rhythmic beats in any sensory modality, regardless of placement. However, this raises the empirical question of whether such placement of haptics may effect entrainment, or whether it may have other effects. We will return to these issues in the discussion section.

Issues concerning sensitivity and intensity also raised questions Rhythms need to be provided with sufficient intensity to allow participants to sense and entrain to them. The vibrotactile intensity therefore had to be strong enough to be readily felt, but gentle enough to be comfortable and pleasant for extended periods of time. Unsurprisingly, the levels required to achieve this balance varied considerably between some of the participants. Most participants preferred the intensity levels at about $40 \%$ intensity. However, P1 (discussed above) had lost $75 \%$ sensitivity from his affected leg, and so requested maximum intensity of haptic cue to allow him to feel able to feel haptic the signal in that leg. Aside from P1, who eventually opted to use a single bracelet, all participants reported finding a suitable balance in intensity after appropriate adjustment.

\section{B. Reactions to the haptic cues}

After trying out the haptic metronome all participants agreed that it gave them a rhythm to walk to. As P4 said, "The beat (rhythm) is, it's something to listen to. [...] the rhythm is good for me."

An interesting observation came from the comments of one participant, P1. Before having a stroke, he was in the army. During the interview session he talked in detail how the rhythmic haptic cue he felt on his leg reminded him of marching in the army. "I remember being in the Army. [...] even in your sleep could do it (marching). But now it's been a long time. It comes back to me. [...] I thought, hang on, me in the Army, doing it, and then it shut up (mentally blocked out) everyone there, that one comes up. 1, 2, 3 and that thing goes 1, $2,3 \ldots$.. (P1). The rhythmic cueing seemed to wake up a long lost memory and helped him "march" in rhythm; "my mind's coming back from the army"'(P1).

Another effect that was observed was the rhythm staying in the participants' head long after the haptic metronome was switched off. All four participants mentioned this phenomenon. "If it is switched off [...], it's still there. [...] in my head" (P4). These observations suggest that participants were readily able to entrain mentally.

\section{Language used}

Participants in this study frequently used "hear", "listen" and other audio related descriptors to describe feeling the felt vibrotactile cues delivered by the haptic metronome. For example, $\mathrm{P} 4$, stated: "The beat is, it's something to listen to". These terminological habits have been noted elsewhere in speech about felt, rather than heard haptic, cues [9].

\section{Design suggestions from participants}

The interview session at the end sparked discussions with stroke survivors on what features improved devices should have and how they should look.

One point arising from all interviews we conducted was the participants' scepticism of using an unaltered beat, or indeed any regular beat on uneven surfaces and badly maintained pavements. All participants agreed that it would be very difficult to maintain a rhythm while negotiating a "difficult" walking surface. The option of having a way to manually adjust cadence settings in such situations was quickly dismissed as being too difficult. Participants commented that the mental burden is already too high while walking without trying to maintain a rhythm. Having to calculate adjustments would only add to that burden. One participant commented that she had only recently trained herself in walking and talking at the same time $(\mathrm{P} 2)$.

The only universally accepted option was to be able to switch the metronome off whenever they felt like it was unsuitable or too difficult to follow safely. The option of making the system "more clever" to self adjust was also considered but we are not sure how well participants really understood the degree to which the devices could reasonably be self-adjusting.

Other design issues considered included the device's size and conspicuousness. A small, light size was felt extremely important, as users would be wearing them for extended periods of time. Also the majority (3/4) of participants agreed that they wanted the devices to be as inconspicuous as possible. P3 notes: "You don't want to feel that it's something that people will stare at you because you've got these things on".

Finally, stroke often causes survivors to lose control of the arm on their paretic side. That means they may experience difficulties in strapping the devices on their legs, therefore special considerations need to be taken when designing the straps.

\section{E. Attitudes of participants}

All four participants indicated that their motivation for participation was to help recent or future stroke survivors: as chronic survivors (see table 1), they appeared dubious about the 
likelihood of any intervention improving their own condition. For example, P2 noted: "Maybe earlier, maybe, I don't know, because I've had my stroke for such a long time, I don't know." Interestingly, the same participant later described how she had recently re-learned how to walk on sand. "[...] I've just learnt how to walk on sand. You know, that's, I've learnt and adapted myself to walking on sand. [... I learned] new strategies [...] a few years ago I couldn't walk on grass and now I can slowly." Thus, despite expressing pessimistic views, there was evidence of learning to adapt to new situations and developing improved strategies for walking.

\section{F. Observations by participants on everyday difficulties}

The interview session at the end of the walking trials provided rich information regarding difficulties faced in every day life in walking after a stroke.

As already noted, the unevenness of the pavements was a common practical problem mentioned by all four participants. "Where I live there's nothing flat" (P1). Having to walk on uneven surfaces is very important for people who already have to put a great amount of effort to coordinate their legs and walk. "I have to think about the pavements" (P2). [...] Instead of looking ahead to the distance you are constantly watching where you are putting your feet you know, more conscious of that factor. (P3)

Participants noted that the situation is made worse when they have to cross the road or when they carry things (e.g. groceries), having to actively shift their balance on one side to compensate. The above points have design implications, as considered in the discussion section below.

\section{DISCUSSION}

Several design issues emerged from consideration of the demands of walking over busy, badly maintained and uneven pavements, in conditions that may be cold or windy. Since we are interested in designing for haptic cueing outside the lab, the identification of such issues is important.

For example, one finding, noted earlier, related to the importance of a quick and simple on/off switch. Previous iterations of the Haptic Bracelets featured large and prominent on/off buttons, and some designs have featured a single such button to switch on and off all bracelets worn by a given user. The present iteration does not currently have such a feature, and the study has made it clear that the use of such a feature could be complicated by problems associated with paretic arms, or when carrying heavy shopping. It may be that voice control, or gestural options, or some kind of auto switch off need to be considered

A related and unexpected finding noted earlier was the unanimous rejection of an option for users to adjust the pace of the metronome dynamically themselves. By contrast, a system able to autonomously adjust tempo to suit varying terrain and conditions was considered highly desirable. Designing such a system would be challenging, but merits serious examination.

Unsurprisingly, different participants preferred different absolute vibrotactile intensities, ranging from around $40 \%$ intensity to $100 \%$ intensity. However, more interesting issues emerged concerning the balance of intensities between the paretic and non-paretic legs. For example, in his initial attempts, participant $\mathrm{P} 1$ preferred very disparate vibrotactile intensities for his two legs, due to a $75 \%$ loss of sensitivity in the affected leg. One might expect that such a disparity could be addressed simply by turning up the vibrotactile intensity on the affected leg. However, after P1's initial attempt to work with a vibrotactile on each leg, he said that he found it difficult to switch attention between legs fast enough and chose to switch to a single vibrotactile on the paretic leg. This raises several interesting issues. Where entrainment has been established, conscious attention is not generally required to be aware of the timing of the next beat. The situation is different when entrainment is in the process of being established, and of course in the case of stimulus response. However, the use of haptic cues to direction attention, or to focus proprioception as possible avenues for influencing gait merits investigation.

Due to P1's aphasia, it was not possible to clarify his observation fully. For example, it would be useful to distinguish between issues of attention switching vs. possible sensory issues. Certainly, especially for any situation in which a limb has lost much of its sensitivity, this issue deserves closer study.

The participants all had some signs of aphasia. This had not previously been considered as having relevance to design issues for the Haptic Bracelets, but may be related to the above issues concerning the directing of sensory attention, which may have design implications for the number, placement and intensity of vibrotactile devices.

One interesting design issue is an apparent tension between the notion of entrainment, and the notion of stimulus response, as noted earlier. Some ramifications of this tension did not become apparent until reflecting on disparate findings of the study.

Previous studies in the audio cueing of gait such as [14], and the earlier study of haptic cueing for gait rehabilitation [8], both discussed earlier, strongly suggest that entrainment is central to metronomic gait rehabilitation. For example in the haptic cueing study [8], which used procedures similar to those of numerous previous audio cueing studies, the participant was instructed to "time her footfalls to the cue".

By contrast, in the more exploratory present study, with the explicit aim of eliciting views from end users and professionals from several disciplines, the deliberate openness of the instructions seems to have been interpreted by participants in ways different from the stricter procedures used in [8]. For example, it is not clear from the motion capture recordings in the present study that participants' footfalls were generally synchronised to the haptic cues. This may have been due primarily to disruptions to the gait caused by the missing cover on the force plate in the floor of the lab (see Limitations section below).

The case of participant P1 raised other issues concerning entrainment. As previously noted, alone out of the participants, P1 preferred to wear a single vibrotactile to help him focus on his paretic leg. Crucially, P1 was provided with only the cue for the paretic leg (without merging the cue signal for the other leg as well). This made complete sense given P1's wish to concentrate on his paretic leg alone, but could act to weaken 
entrainment. Aspects of this deployment of cues contrast with the approach in the earlier study [8], where it also happened that only a single vibrotactile was worn, but the cues for both limbs were directed to the single vibrotactile. Note that for purposes of establishing entrainment, this latter arrangement is unremarkable - as can be seen by analogy with the operation of a simple audio metronome. Indeed, the neuroscientists involved in [8] discussed locating haptic cues anywhere they could most easily be felt and entrained to - including on hips. By contrast with this prioritising of entrainment in [8], in the present study, both physiotherapists, and three of four participants preferred to place a vibrotactile specifically on each leg.

Before leaving the case of P1, note that the cue for the unpaired leg had a period of 1.5 seconds (or 40 beats per minute). This is slow enough to make entrainment challenging, though not impossible.

As previously noted, by contrast to the above entrainmentthemed considerations, the physiotherapists in the present study chose specifically to locate the vibrotactiles on the Tibialis Anterior muscle near the knee, spurred by the idea that timely action might be stimulated, which seems to suggest the idea of stimulus response. This seems to have been motivated in part by physiotherapists' use of the touch of their hands to assist with gait rehabilitation, which may centre on issues other than precise timing. The choice of placement may also have been motivated in part by analogy with FES (Functional Electrical Stimulation) devices, which are used by some hemiparetic patients (though none in the present study). These devices typically employ a sensor worn on the base of the foot to instruct the FES to directly stimulate the relevant muscle on each step to contract the foot to avoid "foot drop "which can otherwise cause stumbling. However, the FES does not give a metronomic cue, rather it is tied directly to the wearer's steps, whenever they may occur.

Despite all the above considerations, at least three participants explicitly noted that the rhythm would remain in their heads for some time after the haptic metronome was switched off. Participants said they could still "hear it" in their head and that it gave them "something to listen to" while walking. Perhaps most interestingly, P1, despite the several potential obstructions to establishing entrainment noted above, said that the haptic metronome woke forgotten memories of marching in the army, and that this helped him maintain a rhythm while walking.

It may well be that for different aspects of rehabilitation, both entrainment and stimulus-response are valuable. Physiotherapists and interaction designers, together with stroke survivors are likely to benefit from working together to understand how these approaches relate, and which are most relevant in what circumstances.

\section{LIMITATIONS}

At the time of the experiment, a force plate (for measuring the impact of steps) was in the process of being installed in the lab, but its cover was not yet fitted, exposing the plate surface, which lay at a lower level than the rest of the floor. Due to the shape of the lab, participants could not easily avoid stepping on the plate and were forced to adjust their gait to step on the plate squarely. This did not impede the business of the user-centred design study, but it did tend to disrupt reliable entrainment, so it was inappropriate to use the present study to gather data on effect of entrainment on gait.

\section{IMPLICATIONS FOR FUTURE DESIGNS AND EXPERIMENTS}

The study has helped to identify numerous design issues as itemised in the Findings and Discussion sections. The study also helped identify several promising directions for future studies. For example, the design tensions between vibrotactile placement and entrainment vs. stimulus response, and the related "stay in head effect" deserve careful further investigation.

A further promising direction for future studies arises from an observation by a physiotherapist after the sessions. Namely, it can be hard to make lasting improvements to the gait of a chronic stroke survivor; however, a period of intensive therapy can temporarily result in substantial improvements. With continued intensive coaching, such gains can be retained, but they are liable to fade as old habits reassert themselves. However, continued extensive coaching is very expensive. This gave rise to the following design proposal. By using a machine learning system in conjunction with the motion sensors in the Haptic Bracelets, the signatures of both the improved gait and the baseline gait could reasonably be recognised. Signatures in between the two extremes could be judged by a process of interpolation. Following an intensive therapy session, such a system could act to some degree as a coach outside the lab for a stroke survivor by monitoring the gait and giving reminders when good habits seemed to be sliding.

\section{CONCLUSIONS}

A blended user-centred design study has been carried out with stroke survivors and diverse professionals on the Haptic Bracelets, a wearable haptic system for gait rehabilitation following hemiparetic stroke. The study identified a wide range of issues with implications for design at disparate levels of specificity and abstraction. Specific findings included: unexpected views from stroke survivors on the desirability of explicit tempo control; an identification of the challenges implicit in designing an on-off switch for two leg-worn bracelets in a single action executable in conditions that are cold wet and windy, and which remains simple-to-use when carrying loads and possessing only a single controllable arm; issues concerning the placement and intensity of vibrotactiles; and issues related to attention and perception when the legs have a major imbalance in the ability to sense touch. Wider findings include identification of a tension between the notion of entrainment and the notion of stimulus response in the design of wearable gait rehabilitation systems. More generally, pervasive healthcare systems need to take into account the physical, sensory and cognitive abilities of intended users. We have demonstrated that a useful way to address such challenges is via a blended user-centred approach with prototypes that allow users to informally reflect and engage. This approach also benefits designers by providing detailed insights and sources of inspiration for future pervasive health designs. 


\section{REFERENCES}

[1] World Health Organization, "15: Global burden of stroke," in The Atlas of heart disease and stroke section. Brighton, United Kingdom: WHO Press, 2004, pp. 50-51.

[2] N. Townsend, K. Wickramasinghe, P. Bhatnagar, K. Smolina, M. Nichols, J. Leal, R. Luengo-Fernandez, and M. Rayner, Coronary heart disease statistics 2012 edition. London: British Heart Foundation, 2012.

[3] J. Adamson, A. Beswick, and S. Ebrahim, "Is stroke the most common cause of disability?," Journal of Stroke and Cerebrovascular Diseases, vol. 13, no. 4, pp. 171-177, July 2004.

[4] Stroke Association. (2013, January) Stroke Statistics. [Online] http://www.stroke.org.uk/sites/default/files/Stroke\%20statistics.pdf

[5] R. Galvin, T. Cusack, and E. Stokes, "To what extent are family members and friends involved in physiotherapy and the delivery of exercises to people with stroke?," Disability and Rehabilitation, vol. 31, no. 11 , pp. $898-905,2009$

[6] J. van der Linden, V. Waights, Y. Rogers, and C. Taylor, "A Blended Design Approach for Pervasive Healthcare: bringing together users, experts and technology," Health Informatics, vol. 18, no. 3, pp. 212-218, September 2012.

[7] S. Holland, R. L. Wright, A. Wing, T. Crevoisier, O. Hödl, and M Canelli, "A gait rehabilitation pilot study using tactile cueing following hemiparetic stroke," in Proceedings of the 8th International Conference on Pervasive Computing Technologies for Healthcare, Oldenburg, Germany, 2014, pp. 302-405.

[8] S. Holland, R. L. Wright, A. Wing, T. Crevoisier, O. Hödl, and M Canelli, "A pilot study using tactile cueing for gait rehabilitation following stroke," in Technology applied to Patient Rehabilitation, Victor M.R. Penichet, Daniyal M. Alghazzawi Habib M.Fardoun, Ed. London, United Kingdom: Springer, 2015, pp. 226-239.

[9] A. Bouwer, S. Holland, and M. Dalgleish, "The Haptic Bracelets: learning multi-limb rhythm skills from haptic stimuli while reading.," in Music and Human-Computer Interaction, S. Holland, K. Wilkie, P. Mulholland, and A. Seago, Eds. London, United Kingdom: Springer, 2013, pp. 101-122.

[10] S. Holland, A. J. Bouwer, M. Dalgelish, and T. M. Hurtig, "Feeling the beat where it counts: fostering multi-limb rhythm skills with the haptic drum kit.," in Tangible, embedded, and embodied interaction, New York, 2010, pp. 21-28

[11] M. H. Thaut, A. K. Leins, R. R. Rice, H. Argstatter, G. P. Kenyon, G. C. McIntosh, H. V. Bolay, and M. Fetter, "Rhythmic auditory stimulation improves gait more than NDT/Bobath training in near-ambulatory patients early poststroke: a single-blind, randomized trial.," Neurorehabil Neural Repair, vol. 21, no. 5, pp. 455-459, 2007

[12] M. Roerdink, C. J. C. Lamoth, G. Kwakkel, P. C. W. Wieringen, and P. J. Beek, "Gait coordination after stroke: benefits of acoustically paced treadmill walking," vol. 87, no. 8, pp. 1009-1022, 2007.

[13] S. Prassas, M. Thaut, G. McIntosh, and R. Rice, "Effect of auditory rhythmic cueing on gait kinematic parameters of stroke patients," Gait \& Posture, vol. 6, no. 3, pp. 218-223, 1997.

[14] R. L. Wright, A. Masood, E. S. MacCormac, D. Pratt, C.M. Sackley, and A.M. Wing, "Metronome-cued stepping in place after hemiparetic stroke: Comparison of a one- and two-tone beat," ISRN Rehabilitation, vol. 2013, 2013.

[15] T. A. Pelton, L. Johannsen, H. Y. Chen, and A. M. Wing, "Hemiparetic stepping to the beat: asymmetric response to metronome phase shift during treadmill gait," Neurorehabilitation and Neural Repair, vol. 24, no. 5, pp. 428-434, 2010.

[16] M. Clayton, R. Sager, and W. Udo, "In time with the music: The concept of entrainment and its significance for ethnomusicology," European meetings in ethnomusicology, vol. 11, pp. 1-82, January 2005.

[17] S. J. Olney, "Hemiparetic gait following stroke. Part I: Characteristics," Gait \& Posture, vol. 4, no. 2, pp. 136-148, April 1996.
[18] C. K. Balasubramanian, R. R. Neptune, and S. A. Kautz, "Variability in spatiotemporal step characteristics and its relationship to walking performance post-stroke," Gait \& Posture, vol. 29, no. 3, pp. 408-414, April 2009.

[19] G. Chen, C. Patten, D. H. Kothari, and F. E. Zajac, "Gait differences between individuals with post-stroke hemiparesis and non-disabled controls at matched speeds," Gait \& Posture, vol. 22, no. 1, pp. 51-56, August 2005.

[20] C. M. Kim and J. J. Eng, "The Relationship of Lower-Extremity Muscle Torque to Locomotor Performance in People With Stroke," Physical Therapy, vol. 83, no. 1, pp. 49-57, January 2003.

[21] D. C. Norvell, J. M. Czerniecki, G. E. Reiber, C. Maynard, J. A. Pecoraro, and N. S. Weiss, "The prevalence of knee pain and symptomatic knee osteoarthritis among veteran traumatic amputees and nonamputees," Archives of Physical Medicine and Rehabilitation, vol. 86, no. 3, pp. 487-151, 2005.

[22] L. Nolan, A. Wit, K. Dudziñski, A. Lees, M. Lake, and M. Wychowañski, "Adjustments in gait symmetry with walking speed in trans-femoral and trans-tibial amputees," Gait \& Posture, vol. 17, no. 2, pp. 142-151, April 2003 .

[23] S. Pouwels, A. Lalmohamed, P. Souverein, C. Cooper, B. J. Veldt, H. G. Leufkens, A. de Boer, T. van Staa, and F. de Vries, "Use of proton pump inhibitors and risk of hip/femur fracture: population-based case-control study," Osteoporosis International, vol. 22, no. 3, pp. 903-910, 2011.

[24] C. L. Richards, F. Malouin, S. Wood-Dauphinee, J. I. Williams, J. P. Bouchard, and D. Brunet, "Task-specific physical therapy for optimization of gait recovery in acute stroke patients.," Archives of Physical Medicine and Rehabilitation, vol. 74, no. 6, pp. 612-620, 1993.

[25] P. J. M. Bank, M. Roerdink, and C. E. Peper, "Comparing the efficacy of metronome beeps and stepping stones to adjust gait: steps to follow!," Experimental Brain Research, vol. 209, no. 2, pp. 159-169, March 2011.

[26] S. Badarny, J. Aharon-Peretz, Z. Susel, G. Habib, and Y. Baram, "Virtual Reality Feedback Cues for Improvement of Gait in Patients with Parkinson's Disease," Tremor Other Hyperkinetic Movement, vol. 4, no. 225, April 2014.

[27] V. Angelis, S. Holland, M. Clayton, and P. J. Upton, "Testing a computational model of rhythm perception using polyrhythmic stimuli," Journal of New Music Research, vol. 42, no. 1, pp. 47-60, March 2013.

[28] J. Phillips-Silver, C. A. Aktipis, and G. A. Bryant, "The ecology of entrainment: foundations of coordinated rhythmic movement," Music Perception, vol. 28, no. 1, pp. 3-14, September 2010.

[29] T. Igoe, O, and D. Sulivan, Physical Computing: Sensing and Controlling the Physical World.: Premier Press, 2004.

[30] S. R. Klemmer, B. Hartmann, and L. Takayama, "How bodies matter: five themes for interaction design," in Proceedings of the 6th conference on Designing Interactive systems, Pennsylvania, 2006, pp. 140-149.

[31] Y. Rogers et al., "Things aren't what they seem to be: innovation through technology inspiration," in Proceedings of the 4th Conference on Designing Interactive Systems: Processes, Practices, Methods, and Techniques, London, 2002, pp. 373-378.

[32] R. McCarney, J. Warner, S. Iliffe, R. van Haselen, M. Griffin, and P. Fisher, "The Hawthorne Effect: a randomised, controlled trial," BMC Medical Research Methodology, vol. 7, no. 30, July 2007. 